\title{
Infection dynamics of digital dermatitis in first-lactation Holstein cows in an infected herd
}

\author{
N. Capion, ${ }^{\star 1}$ M. Boye, † C. T. Ekstrøm, $\ddagger$ and T. K. Jensen† \\ *Department of Large Animal Sciences, Faculty of Life Sciences, University of Copenhagen, 2630 Taastrup, Denmark \\ †National Veterinary Institute, Technical University of Denmark, 1870 Frederiksberg, Denmark \\ $\ddagger$ Department of Biostatistics, University of Southern Denmark, 5000 Odense, Denmark
}

\begin{abstract}
Digital dermatitis (DD) refers to painful lesions primarily affecting the skin in the interdigital region of dairy cattle. The purpose of this study was to evaluate the dynamics of DD in 39 cows, observed at approximately 3 -d intervals, for the first 6 mo of lactation. Specifically, the study aimed at evaluating different levels of DD susceptibility in cows, identifying the bacterial colonization of the interdigital skin, and exploring the relationship between clinical DD diagnosis and laboratory findings. Three different susceptibility categories were identified for DD: $1=$ consistently healthy cow; $2=$ intermittently infected cow; and $3=$ consistently infected cow. Susceptibility categories were associated with age at calving. The average age at calving was 775 $\mathrm{d}(\mathrm{SD} \pm 43.4)$, with the youngest heifer calving at age $669 \mathrm{~d}$ and the oldest heifer at $858 \mathrm{~d}$. Advancing age at calving was associated with greater odds of being intermittently or consistently infected. This corresponded with an odds ratio of 2.02 over a period of $30 \mathrm{~d}$. During the study period, $161 \mathrm{DD}$ lesions were identified in 28 of the 39 cows $(72 \%)$. Of those 28 cows, 13 cows were consistently infected. The remaining 11 of the 39 cows $(28 \%)$ showed slight thickening of the skin with no pain (5 cows) and no signs of skin changes (6 cows). Histopathology and fluorescence in situ hybridization were possible to perform on 132 biopsy samples. A clinical diagnosis of DD was confirmed in $70 \%$ of the lesions by histopathology, and colonization of Treponema spp. Dichelobacter nodosus was found in 35 samples (29\%).
\end{abstract}

Key words: digital dermatitis, infection dynamics, lameness

\section{INTRODUCTION}

Digital dermatitis (DD) continues to be a worldwide cause of serious painful infectious lesions, lameness, reduced welfare, and production losses in dairy herds

Received January 12, 2012.

Accepted July 5, 2012.

${ }^{1}$ Corresponding author: nyc@life.ku.dk
(Capion et al., 2008; Bruijnis, 2010; Cha, 2010). During the last few decades, the etiology of DD has been elucidated, in which Treponema spp. have been established as the probable causative agent (Evans et al., 2008; Klitgaard et al., 2008; Yano, 2010). However, the current knowledge of the dynamics of DD is not sufficient to recommend effective prevention and control strategies for infected herds. For example, identification of the bacteria or microorganisms that initiate the clinical disease as well as mapping the spread of the disease would be of great value to develop better prevention and control strategies.

Observations have been made by farmers as well as hoof trimmers of cows within endemic DD herds that never develop clinical DD. Some researchers has posed this as a possibility (Laven and Logue, 2007; Holzhauer et al., 2008; Scholey et al., 2011), but it has not yet been evaluated critically. However, investigating these observations with the current knowledge of DD dynamics (Döpfer et al., 2008; Nielsen et al., 2009; Capion, 2011) would require further longitudinal studies. Furthermore, the extrapolation of findings between herds is difficult because multiple risk factors have been shown to affect the incidence of DD (Rodriguez-Lainz et al., 1999; Wells et al., 1999; Holzhauer et al., 2006).

The purpose of this study was (1) to evaluate the possible presence of different levels of susceptibility in cows and determine whether specific cow factors influence susceptibility; (2) to identify the treponemal colonization of the interdigital skin from the earliest changes in skin biopsies collected during the course of DD lesions in 39 heifers followed intensely for the first 6 mo of lactation; and (3) to evaluate the relationship between clinical diagnosis of DD and laboratory findings.

\section{MATERIALS AND METHODS}

In a commercial Danish Holstein dairy herd with 350 lactating cows, 39 heifers calving from July through October 2009 were selected. They were first examined 2 to 4 wk before calving. Locomotion was scored according to the method of Sprecher et al. (1997), and 
all hind feet were examined and lesions were recorded in a portable trimming chute. Thirty-five cows were observed in approximately 3 -d (2- to 5 -d) intervals during the next 6 mo. Four cows left the study prematurely because of culling and difficult temperament. The skin adjacent to the horn capsule was inspected and palpated while the cows were standing or lying in the cubicle. A score describing changes and pain in the skin was developed: inspection score $0=$ skin looks normal, with no swelling and no pain or soreness; normal smooth, soft skin; 1 = skin looks irritated; swelling may be present, with no pain or soreness; skin is rough and hard on palpation; and $2=$ skin looks irritated and changes are visible, with a marked line between normal and changed skin; pain or soreness on palpation; the skin feels moist or rough on the surface. Cattle with skin changes (inspections scores of 1 and 2) were examined further in the trimming chute. After removing manure and debris, the lesions were photographed and scored macroscopically using the M-scale (Döpfer et al., 1997), where M0 denotes normal skin and M1, M2, M3, and M4 denote different stages of DD. Skin biopsies were taken to the level of the basal membrane, cutting with a 6-mm punch biopsy (Kruuse, Odense, Denmark) but parallel to the skin surface from lesions with inspection score 2 and lesion stages M1, M2, M3, and M4. Before sampling, the skin was anesthetized using ice spray (Prof Care, Ice Spray; Select Sports, Glostrup, Denmark). Samples were collected from lesions (M1, M2, M3, and M4) every third day until either the lesions resolved (inspection score of 0 or 1 ) or the pain level reached the preset threshold (grade 4 lameness; Sprecher et al., 1997), when treatment was initiated. Treatment consisted of a bandage with salicylic acid powder for $2 \mathrm{~d}$.

Biopsies were fixed in 10\% neutral buffed formalin, dehydrated, and embedded in paraffin blocks. Serial tissue sections $(4 \mu \mathrm{m})$ were cut and mounted on SuperFrost+ slides (Menzel-Gläser, Braunschweig, Germany) for hematoxylin and eosin staining and fluorescence in situ hybridization (FISH).

\section{Histopathological Evaluation}

All samples were evaluated histopathologically in hematoxylin and eosin staining by 1 researcher (T.K.J.). Epidermal changes were scored from 0 to $4(0=$ normal epidermis; $1=$ hyperkeratosis and epidermal hyperplasia; $2=$ focal; $3=$ moderate; and $4=$ severe acanthotic epidermis with or without parakeratotic papillomatous proliferation and ballooning degeneration of keratinocytes, exocytosis, or erosion of dermal papilla, or their combination).

\section{FISH}

The biopsies were hybridized using oligonucleotide probes specific for the domain Bacteria, Dichelobacter nodosus, different Treponema spp. phylotypes, and a Treponema group probe. The Treponema spp. phylotypes investigated in this study were the 10 most prevalent ones according to Klitgaard et al. (2008; PT1, PT2, PT4, PT5, PT6, PT7, PT8, PT9, and PT17, where PT refers to phylotypes). In brief, the oligonucleotide probes were $5^{\prime}$ labeled with fluorescein isothiocyanate or $\mathrm{Cy} 3$ dye. Hybridization was carried out at $46^{\circ} \mathrm{C}$ with hybridization buffer $(100 \mathrm{~m} M$ Tris, $\mathrm{pH} 7.2,0.9 \mathrm{M} \mathrm{NaCl}, 0.1 \%$ SDS) containing $5 \mathrm{ng} / \mu \mathrm{L}$ of each oligonucleotide for $16 \mathrm{~h}$ in a Sequenza slide rack (Thermo Shandon, Cheshire, UK). Slides were then washed with prewarmed $\left(46^{\circ} \mathrm{C}\right)$ hybridization buffer for $3 \times 3 \mathrm{~min}$, followed by wash with prewarmed $\left(46^{\circ} \mathrm{C}\right)$ washing buffer (100 $\mathrm{m} M$ Tris, $\mathrm{pH} 7.2,0.9 \mathrm{M} \mathrm{NaCl})$ for $3 \times 3 \mathrm{~min}$. Finally, they were rinsed in water, air-dried, and mounted with Vectashield (Vector Laboratories Inc., Burlingame, CA) for epifluorescence microscopy. An Axioimager M1 epifluorescence microscope was used, and images were obtained using an AxioCAM MRm version 3 FireWiremonochrome camera (Carl Zeiss, Oberkochen, Germany).

The hybridized sections were all read and scored by 1 researcher (T.K.J.). The total bacterial (probe for the domain Bacteria) invasion of the epidermis was scored from 0 to 3 (i.e., $0=$ no invasive bacteria; $1=$ low grade of invasive bacteria; $2=$ moderate grade of invasive bacteria; and $3=$ high grade of invasive bacteria). The prevalence of the general Treponema probes and each of the Treponema phylotypes was scored from 0 to $3(0=$ no invasive treponemes; 1 = low grade of treponemes; 2 $=$ moderate grade of treponemes; and $3=$ high grade of treponemes; Klitgaard et al., 2008), whereas the prevalence of $D$. nodosus was scored as $0=$ no hybridization, or $1=$ positive hybridization.

\section{Statistical Analyses}

Statistical analysis of the disease dynamics was performed on data from the 35 cows that completed the study, using $k$-means clustering, hierarchical clustering, and multivariate methods (e.g., principal components analysis) to identify parameters that possibly corresponded to different susceptibility categories (i.e., 1 $=$ consistently healthy; $2=$ intermittent DD; and 3 $=$ consistently DD). Sensitivity and specificity of the resulting categories were estimated. A generalized estimating equation (GEE) approach, which allows for repeated measurements, was used for an ordinal regres- 
sion model to test if the age at calving influenced the susceptibility category.

Cluster Identification. Only lesions that received an inspection score of 2 (changes and pain) were confirmed by clinical examination, microbiology, and histopathology. Consequently, the cows were examined in the trimming chute and biopsy sampled with slightly different frequencies and over different intervals. An estimate was made of the smoothed average profile over time since calving, based on all inspection scores. Each individual cow (and possible leg) was compared with the average cow profiles to identify groups that differed.

The clustering was based solely on the inspection score. Different features were used to identify groupings in the data. The 10 criteria listed below were selected because they capture various facets of the data. Criterion 1 and criterion 2 deal with data measurement frequency, whereas criterion 3 to criterion 10 try to address different types of deviations from the "average" profile of a cow. For each cow, the following 10 features were used:

1. the total number of observations;

2. the proportion of the total range of time since calving that was covered by observations from the cow;

3. the average difference for each observation to the average population score for each exam time;

4. the average squared difference for each observation to the average population score for each exam time;

5. the difference in area under the curve between the actual inspection score compared with the area under the curve for the average population relative to the proportion of the range covered;

6. the squared difference in area under the curve between the actual inspection score compared with the area under the curve for the average population relative to the proportion of the range covered;

7. the difference in area under the curve between the observed inspection curve and the average population in the early period (see below);

8. the squared difference in area under the curve between the observed inspection curve and the average population in the early period (see below);

9. the difference in area under the curve between the observed inspection curve and the average population in the late period (see below); and

10. the squared difference in area under the curve between the observed inspection curve and the average population in the late period (see below).
The time since calving was split into two regions, termed "early" and "late," to see if the changes could be assigned to 1 of the 2 periods. The cutoff point was chosen arbitrarily at $50 \mathrm{~d}$.

For the initial exploratory data analysis, we considered a principal components regression on the raw feature measurements. However, because the features were on vastly different scales (e.g., we were comparing number of sample times for each leg to sample range to the squared difference of the observed inspection profiles to the average cow profile), we decided to normalize the values for each of the 10 different features to make the numerical values more comparable.

Susceptibility Category and Age at Calving. A generalized estimating equation model was used to analyze the association between age at calving and disease category. The disease category was used as an ordered response, and age at calving was used as an explanatory variable.

For the GEE procedure, a multinomial error distribution was specified as well as a cumulative logistic link function with a working independence covariance structure with clusters based on cows. In the initial model, age and age squared were included as explanatory variables.

Laboratory Measurements. Registered measurements were used for the prevalence of invasive Treponema phylotypes and epidermal changes seen, to identify if any linear trends existed in the profiles for the 2 variables. For these analyses, data from all 39 cows were used. Note that we had very few observations, so any detailed effects were not possible to ascertain. For each response variable (e.g., prevalence of Treponema and epidermal changes), GEE was used with an ordinal regression model to test whether the time since calving was associated with the response.

\section{RESULTS}

In total, 39 Holstein heifers were selected that calved from July 5, 2009, through October 2009. These heifers were born between June 1, 2007, and November 7, 2007. In 4 cows, fewer than 6 observations were possible because of culling or difficult temperament, and in the remaining 35 cows, an average of $30(\mathrm{SD} \pm 10.2)$ observations were made per cow from September 7, 2009, until March 11, 2010. The average age at calving was $775 \mathrm{~d}$ (SD \pm 43.4 ), with the youngest heifer calving at age $669 \mathrm{~d}$ and the oldest heifer calving at $858 \mathrm{~d}$.

Before calving, 1 heifer received an inspection score of 1 on 1 leg, 1 heifer received an inspection score of 2 on $1 \mathrm{leg}$, and 1 heifer received an inspection score of 2 on both hind legs. During the study, 472 legs, for a total of 3,288 inspected legs, received an inspection score of 


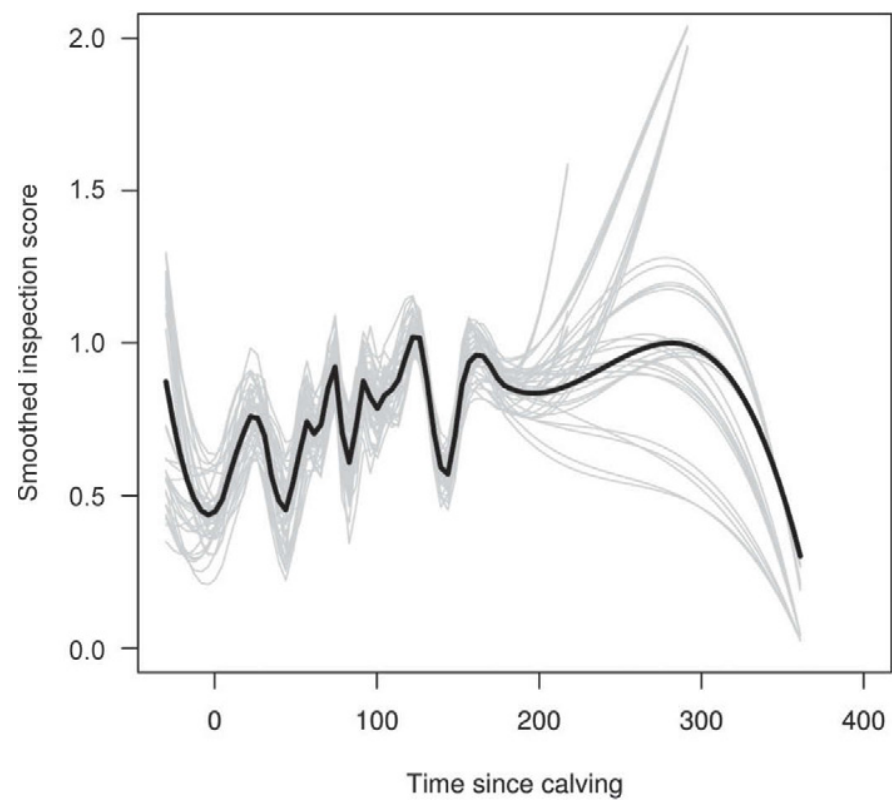

Figure 1. Average inspection score profile as a function of time since calving (black) with individual inspection score profiles overlaid (gray).

1 , and only 3 of those were scored as DD (all M1) at examination in the trimming chute. Seventy-two percent of the cows had 1 or more cases of DD (M1, M2, M3, or M4) during the study period. Eleven cows (28\%) never showed clinical changes in the skin around the claws.

During the study, a total of 161 legs received an inspection score of 2 . Of those, $74(46 \%)$ had M1 lesions, 75 (47\%) had M2 lesions, 2 (1\%) had M3 lesions, and $10(6 \%)$ had M4 lesions. The first M2 lesions were observed $9 \mathrm{~d}$ after calving. The first M4 lesions (chronic DD) were seen 10 wk after calving. Eight M4 lesions followed the M2 lesions, 1 M4 lesion followed an M3 lesion, and 1 followed an M1 lesion.

\section{Susceptibility Categories}

Sampling frequency differed among cows. Typically, the observation frequency in the trimming chute increased for individuals with high inspection scores. Some cows or legs had only a single measurement or a few measurements; thus, a category was difficult to predict for those individuals.

Cluster Identification. Figure 1 shows the average smoothed inspection score profile as a function of time since calving. Generally, it appeared as if the 3 variables (i.e., mean area under the curve difference, squared area under the curve difference, and late mean area under the curve difference) provided a good segmentation of the data.
The $k$-means clustering groups showed the identifying features of the groups. Susceptibility category 1 generally had low profiles and a low late profile, whereas susceptibility category 2 had a slightly higher profile closer to the average profile and susceptibility category 3 had a high-level profile. For 22 cows, the analysis placed both hind legs in the same category: 11 cows were consistently healthy in both hind legs, 4 were intermittently infected in both hind legs, and 7 were consistently infected in both legs.

In 13 cows, this was not the case. Six cows had 1 leg in the consistently healthy category and the other leg was in the intermittently infected category. For 7 cows, $1 \mathrm{leg}$ was in the intermittently infected category and the other leg was in the consistently infected category. Four cows had too few observations to estimate the category.

A linear discriminant analysis was used to check the stability of the categorization based on the chosen features (Figure 2). The 3 different categories (labeled 1, 2 , and 3 ) were segmented nicely into 3 groups, indicating that the choice of features partitioned the observations into categories quite effectively. The proportion of observations classified in the same way as for $k$-means clustering when using linear discriminant analysis was 0.97 .

Effect of Susceptibility Category and Cow Level Risk Factors. Age at calving squared did not have a significant effect $(P=0.436)$, and age at calv-

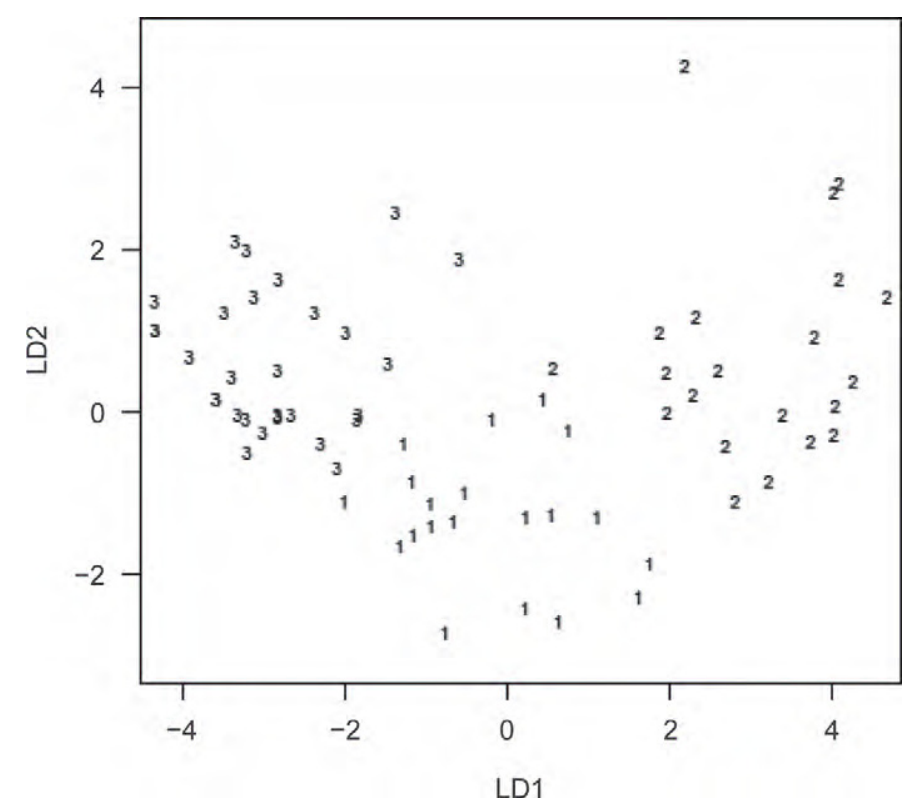

Figure 2. Results of the linear discriminant analysis. Linear discriminant 1 (LD1) is the first linear discriminator and linear discriminant 2 (LD2) is the second linear discriminator. The chosen feature partitioned the observations into categories quite effectively. 
Table 1. Distribution between the total bacterial invasion of the epidermis and the prevalence of invasive Treponema phylotypes detected and quantitatively scored by fluorescence in situ hybridization in digital dermatitis lesions

\begin{tabular}{lrrrrr}
\hline & \multicolumn{5}{c}{ Prevalence of invasive Treponema phylotypes } \\
\cline { 2 - 6 } Bacterial invasion & No & Low & Moderate & High & Total \\
\hline No bacteria & 32 & 0 & 0 & 0 & 32 \\
Low number & 8 & 11 & 0 & 0 & 19 \\
Moderate number & 0 & 3 & 11 & 1 & 15 \\
High number & 0 & 0 & 0 & 66 & 66 \\
Total & 40 & 14 & 11 & 67 & 132 \\
\hline
\end{tabular}

ing was significant $(P=0.026)$. This meant that each increase in age at calving (measured in days) had an odds ratio of $\exp (0.02)=1.02$ of resulting in a higher susceptibility category and a higher risk of DD. Consequently, the risk of a higher susceptibility category increased with age at calving. This corresponded over a period of $30 \mathrm{~d}$ to an odds ratio of $\exp (30 \times 0.02)=$ 2.02. The $95 \%$ confidence interval for the odds ratio for the effect of each single day was at $(1.00 ; 1.04)$.

\section{Bacterial Invasion and Histopathological Changes}

Histopathology and FISH were performed on 132 biopsy samples of M1, M2, M3, and M4 lesions. From 2 lesions, 4 biopsies were taken before the lesion resolved or the lesion required treatment; from 3 lesions, 3 biopsy samples were taken; from 22 lesions, 2 biopsies were taken; and from 71 lesions, 1 biopsy was taken. Treponema phylotypes were found in 92 out of 132 samples (70\%), and Dichelobacter nodosus was detected in 35 out of 132 samples (27\%). The distribution between the total bacterial load and the prevalence of Treponema was shown in Table 1. In 32 out of 132 samples $(25 \%)$, no bacteria were demonstrated by the general eubacterial probe. These samples were collected from M1-, M2-, and M4-stage lesions.
The 2 phylotypes, PT2 and PT8, were not detected in any of the samples; prevalence of the 7 remaining phylotypes varied within lesions. The distribution was shown in Table 2.

As is evident in Table 2, no Treponema brennaborenselike phylotypes were detected in the lesions. The single most common microorganism detected in lesions in this study was from the Treponema refringens/Treponema calligyrum-like phylogenetic cluster. In the 92 positive lesions, an average of 5.9 phylotypes were present per lesion.

In Table 3, the distribution between the prevalence of Treponema phylotypes and epidermal changes is shown for the 132 biopsies. In 5 samples of normal skin, no Treponema spp. were found. The number of Treponema phylotypes increased with increased histopathological epidermal changes. Microscopically, the biopsies represented epidermal layers of the skin with or without dermal papilla; thus, further characterization of dermal alterations was not possible.

Effect of Time on the Prevalence of Treponema Phylotypes. A test of the linear association between time since calving and the prevalence of Treponema yielded a $P$-value of 0.345 . No definite pattern was observed in the presence in Treponema spp. over time. In addition, no relationship was observed between the

Table 2. Quantitative distribution of different Treponema phylotypes in skin samples of digital dermatitis lesions from cows across the study period

\begin{tabular}{llcccc}
\hline $\begin{array}{l}\text { Treponema } \\
\text { cluster }^{1}\end{array}$ & Probe $^{2}$ & $\begin{array}{c}\text { Total positive } \\
\text { samples }\end{array}$ & $\begin{array}{c}\text { Low number } \\
\text { of treponemes }\end{array}$ & $\begin{array}{c}\text { Moderate number } \\
\text { of treponemes }\end{array}$ & $\begin{array}{c}\text { High number } \\
\text { of treponemes }\end{array}$ \\
\hline 3 & PT1 & 64 & 34 & 24 & 6 \\
3 & PT4 & 66 & 30 & 29 & 7 \\
4 & PT5 & 71 & 40 & 29 & 2 \\
2 & PT6 & 65 & 17 & 39 & 9 \\
1 & PT7 & 62 & 22 & 30 & 4 \\
3 & PT9 & 63 & 34 & 25 & 14 \\
3 & PT17 & 79 & 32 & 33 & 4 \\
\hline
\end{tabular}

${ }^{1}$ According to Yano et al. (2010), the treponemes present in lesions can be categorized in phylogenetic clusters: cluster 1 (Treponema putidum/Treponema pedis/Treponema denticola-like), cluster 2 (Treponema phagedenislike), cluster 3 (Treponema refringens/Treponema calligyrum-like), cluster 4 (Treponema medium/Treponema vincentii-like), and cluster 5 (Treponema brennaborense-like).

${ }^{2} \mathrm{PT}=$ phylotypes. 
Table 3. Distribution between the prevalence of Treponema phylotypes and the histopathological score of epidermal changes ${ }^{1}$

\begin{tabular}{lccccr}
\hline Item & $\begin{array}{c}\text { No } \\
\text { treponemes }\end{array}$ & $\begin{array}{c}\text { Low } \\
\text { grade }\end{array}$ & $\begin{array}{c}\text { Moderate } \\
\text { grade }\end{array}$ & $\begin{array}{r}\text { High } \\
\text { grade }\end{array}$ & Total \\
\hline Normal epidermis & 5 & 0 & 0 & 0 & 5 \\
Hyperkeratosis, hyperplasia & 7 & 5 & 2 & 5 & 19 \\
Focal changes & 8 & 1 & 3 & 8 & 20 \\
Moderate change & 11 & 4 & 4 & 26 & 45 \\
Severe change & 9 & 4 & 2 & 28 & 43 \\
Total & 40 & 14 & 11 & 67 & 132 \\
\hline
\end{tabular}

${ }^{1}$ Scores: $0=$ normal epidermis; $1=$ hyperkeratosis and epidermal hyperplasia; $2=$ focal; $3=$ moderate; and 4 $=$ severe acanthotic epidermis with or without parakeratotic papillomatous proliferation and ballooning degeneration of keratinocytes, exocytosis, erosion of the dermal papilla, or their combination.

time since "infection" and the number of treponemes present in lesions.

Effect of Time on Epidermal Changes. A test of the linear association between time since calving and degree of epidermal changes yielded a $P$-value of 0.995 . This probably occurred because of too much variation in the development of changes within the epidermis over time.

\section{Relationship Between Clinical DD and Laboratory Findings}

No bacteria were detected by FISH in 12 of 51 M2 lesions and in 5 of 9 M4 lesions (Table 4). The FISH detected only a low number of invasive bacteria and no treponemes in 4 lesions scored as M1, 4 scored as $\mathrm{M} 2$, and 5 scored as M4, respectively. In the remaining samples, the DD diagnosis (M1, M2, M3, and M4) was supported by histopathology and by the identification of treponemes. As shown in Tables 3, 4, and 5, in approximately $30 \%$ of the lesions, the clinical diagnosis could not be supported by the laboratory analysis (e.g., histopathology and FISH).

\section{DISCUSSION}

The identification of different levels of susceptibility corresponded well with the proposed findings of consistently healthy cows within endemically infected herds (Laven and Logue, 2007; Scholey et al., 2011). In addition, it made sense that the definition of categories used in this analysis placed some cows in 2 different groups based on lesions found in the left and right hind legs. No cows had legs assigned to category 1 (consistently healthy) and category 3 (consistently infected). Some cows had legs that received scores of 1 and 2 or 2 and 3 ; thus, those cows were classified in either the intermittently infected category or the consistently infected category. Some cows may experience differences in susceptibility between the right and left sides. This may be due to environmental factors, such as the orientation of the feed bunk relative to the direction of the manure scraper, movement within the barn, location of the footbath, or whether the cow has a preferred lying position. One could speculate that skin damaged by DD is more susceptible to new cases during the healing process, which would render that leg more susceptible after the first infection.

The most interesting finding was that some cows, in this case $11(28 \%)$ cows, did not appear to be affected by the environment in the broadest terms of a commercial dairy herd. This group of cows was especially interesting to identify to further explore the reason for this low susceptibility or high resistance toward DD. Some genotypic variation appeared to support this finding (Scholey et al., 2011).

Placing the cows in the different categories was based on the inspection scores done in the herd on soiled legs and on animals standing or lying. This method likely has a rather low specificity; for example, some animals

Table 4. Relationship between the prevalence of Treponema phylotypes in skin samples from digital dermatitis (DD) lesions and the corresponding clinical macroscopic classification of DD lesion stages

\begin{tabular}{lcccrr}
\hline DD stage & $\begin{array}{c}\text { No } \\
\text { treponemes }\end{array}$ & $\begin{array}{c}\text { Low } \\
\text { number }\end{array}$ & $\begin{array}{c}\text { Moderate } \\
\text { number }\end{array}$ & $\begin{array}{c}\text { High } \\
\text { number }\end{array}$ & Total \\
\hline M1 & 23 & 10 & 7 & 31 & 71 \\
M2 & 12 & 3 & 4 & 32 & 51 \\
M3 & 0 & 0 & 0 & 1 & 1 \\
M4 & 5 & 1 & 0 & 3 & 9 \\
Total & 40 & 14 & 11 & 67 & 132 \\
\hline
\end{tabular}


Table 5. Relationship between the histopathological score for epidermal changes ${ }^{1}$ and classification of the clinical stage of digital dermatitis (DD) lesions

\begin{tabular}{lccccc}
\hline DD stage & $\begin{array}{c}\text { Normal } \\
\text { epidermis }\end{array}$ & $\begin{array}{c}\text { Hyperkeratosis, } \\
\text { hyperplasia }\end{array}$ & $\begin{array}{c}\text { Focal epidermal } \\
\text { damage }\end{array}$ & $\begin{array}{c}\text { Moderate epidermal } \\
\text { damage }\end{array}$ & $\begin{array}{c}\text { Severe epidermal } \\
\text { damage }\end{array}$ \\
\hline M1 & 3 & 13 & 14 & 28 & 13 \\
M2 & 2 & 6 & 3 & 0 & 14 \\
M3 & 0 & 0 & 0 & 4 & 1 \\
M4 & 0 & 0 & 3 & 26 \\
\hline
\end{tabular}

${ }^{1}$ Scores: $0=$ normal epidermis; $1=$ hyperkeratosis and epidermal hyperplasia; $2=$ focal; $3=$ moderate; and $4=$ severe acanthotic epidermis with or without parakeratotic papillomatous proliferation and ballooning degeneration of keratinocytes, exocytosis, erosion of dermal papilla, or their combination.

will have skin changes that are not detected. Another method, which could be expected to have a higher specificity, could be to examine all cows in a trimming chute, but more time and labor are needed, and even then, lesions would be missed.

The relationship between a young calving age and low risk of DD can be due to several factors. Intuitively, calves that are healthy throughout their youth reach the intended insemination weight and size sooner (Hultgren and Svensson, 2010; Heinrichs and Heinrichs, 2011). This further supports the emphasis on health management in calves and young stock. One can speculate that different tissue types are associated with different levels of susceptibility toward DD and possibly other diseases involving the epithelium, which would imply that some calves are born healthier than others. Another thought could be that they have a different conformation (e.g., heel height, toe angle, hock angle; Laven, 2007) and thereby are less susceptible to DD, as well as experiencing less lameness caused by other conditions in the claws and legs.

A diagnosis could not be confirmed in $30 \%$ of the lesions classified as the M1, M2, or M4 stages of DD by detection of treponemes with FISH. This is in contrast to a previous study in which the correlation between the clinical diagnosis DD and Treponema was high (40 samples positive out of 41 samples in total; Klitgaard et al., 2008). However, Klitgaard et al. (2008) reported single samples from typical DD lesions (M2 and M4), whereas in the present study, sampling was from all stages of DD or changes in the normal appearance of the skin. Furthermore, the sampling technique chosen for the present study was different, allowing repeated samples of the same lesion over a period of time. In 1 cow, a lesion was classified as M2 when FISH was followed with the general Treponema probe, and no treponemes were found. Nevertheless, with FISH for the PT17 probe, the number of invasive treponemes in the skin was low. A simple explanation is that FISH is performed on many parallel cuts, and if only a few treponemes are present in the biopsy, then it is possible that the general Treponema probe might look negative and one or more of the other probes might be positive. Thus, the relatively poor agreement between clinics and laboratories in $30 \%$ of the lesions may be due to the low sensitivity of FISH. This could be the case especially when only a few treponemes are present, when a poor tissue sampling technique (biopsies taken parallel to the skin instead of perpendicular) is used, or perhaps when certain stages of DD exist or persist without the presence of invasive Treponema or with a very low prevalence of it. Furthermore, the macroscopic or clinical appearance of the lesion could be misclassified.

Because of the highly dynamic nature of the lesions evaluated in this study, it was not possible to show an association between the histopathological changes in the skin, the presence of Treponema spp., and time since calving or time since the first sign of infection. We could, however, show a higher number of and variation in treponemes in M2-, M3-, and M4-stage lesions compared with M1.

\section{CONCLUSIONS}

In total, 39 Holstein heifers were examined for DD for a 6-mo period. During the study, a total of 161 legs with DD were biopsied in $72 \%$ of the cows. Eleven cows $(28 \%)$ never showed clinical changes in the skin around the claws on inspection and palpation of the skin. We identified 3 average profile types that can be used to represent different susceptibility categories for DD: $1=$ consistently healthy cow; $2=$ intermittently infected cow; and $3=$ consistently infected cow. The categories found were associated with age at calving. The higher the age at calving, the greater odds of being in the intermittently infected or consistently infected group. Histopathology and FISH were performed on 132 biopsy samples. Treponema phylotypes were found in 92 samples (70\%), and Dichelobacter nodosus was detected in 35 samples (27\%). The 2 phylotypes, PT2 and PT8, were not detected in any of the samples, whereas prevalence of the 8 remaining phylotypes varied within lesions. The most common microorganism detected was 
from the Treponema refringens/T. calligyrum-like phylogenetic cluster. In $30 \%$ of the lesions, no Treponema phylotypes were detected; consequently, the clinical diagnosis cannot be supported by histopathology and FISH.

\section{ACKNOWLEDGMENTS}

The study was funded by the Danish Research Council for Technology and Production (Copenhagen). We thank the farmers for allowing us on the farm for such a long time.

\section{REFERENCES}

Bruijnis, M. R. N. 2010. Assessing economic consequences of foot disorders in dairy cattle using a dynamic stochastic simulation model. J. Dairy Sci. 93:2419-2432.

Capion, N. 2011. Incubation period for digital dermatitis. Page 13 in Proc. 16th Symp. 8th Conf. Lameness in Ruminants, Rotorua, New Zealand. ConferenceNZ, Rotorua, New Zealand.

Capion, N., S. M. Thamsborg, and C. Enevoldsen. 2008. Prevalence of foot lesions in Danish Holstein cows. Vet. Rec. 163:80-85.

Cha, E. 2010. The cost of different types of lameness in dairy cows calculated by dynamic programming. Prev. Vet. Med. 97:1-8.

Döpfer, D., T. Bennett, and N. Cook. 2008. Dynamics of digital dermatitis infection spread in a large freestall housed Wisconsin dairy herd. Pages 300-301 in Proc. 15th Symp. 7th Conf. Lameness in Ruminants, Kuopio, Finland. Juvenes Print, Tampere, Finland.

Döpfer, D., A. Koopmans, F. A. Meijer, I. Szakall, Y. H. Schukken, W. Klee, R. B. Bosma, J. L. Cornelisse, A. J. A. M. vanAsten and A. A. H. M. terHuurne. 1997. Histological and bacteriological evaluation of digital dermatitis in cattle, with special reference to spirochaetes and Campylobacter faecalis. Vet. Rec. 140:620-623.

Evans, N. J., J. M. Brown, I. Demirkan, R. D. Murray, W. D. Vink, R. W. Blowey, C. A. Hart, and S. D. Carter. 2008. Three unique groups of spirochetes isolated from digital dermatitis lesions in UK cattle. Vet. Microbiol. 130:141-150.
Heinrichs, A. J., and B. S. Heinrichs. 2011. A prospective study of calf factors affecting first-lactation and lifetime milk production and age of cows when removed from the herd. J. Dairy Sci. 94:336341.

Holzhauer, M., D. Döpfer, J. De Boer, and G. Van Schaik. 2008. Effects of different intervention strategies on the incidence of papillomatous digital dermatitis in dairy cows. Vet. Rec. 162:41-46.

Holzhauer, M., C. Hardenberg, C. J. M. Bartels, and K. Frankena. 2006. Herd- and cow-level prevalence of digital dermatitis in the Netherlands and associated risk factors. J. Dairy Sci. 89:580-588.

Hultgren, J., and C. Svensson. 2010. Calving interval in dairy cows in relation to heifer rearing conditions in southwest Sweden. Reprod. Domest. Anim. 45:136-141.

Klitgaard, K., M. Boye, N. Capion, and T. K. Jensen. 2008. Evidence of multiple Treponema phylotypes involved in bovine digital dermatitis as shown by $16 \mathrm{~S}$ rRNA gene analysis and fluorescence in situ hybridization. J. Clin. Microbiol. 46:3012-3020.

Laven, R. A. 2007. The relationship between hoof conformation and digital dermatitis in dairy cattle. Cattle Pract. 15:93-95.

Laven, R. A., and D. N. Logue. 2007. The effect of precalving environment on the development of digital dermatitis in first lactation heifers. Vet. J. 174:310-315.

Nielsen, B. H., P. T. Thomsen, and J. T. Sørensen. 2009. A study of duration of digital dermatitis lesions after treatment in a Danish dairy herd. Acta Vet. Scand. 51:27-31.

Rodriguez-Lainz, A., P. Melendez-Retamal, D. W. Hird, D. H. Read, and R. L. Walker. 1999. Farm- and host-level risk factors for papillomatous digital dermatitis in Chilean dairy cattle. Prev. Vet. Med. 42:87-97.

Scholey, R., B. Ollier, R. Murray, R. Smith, S. Carter, and R. Blowey. 2011. Genetic loci associated with digital dermatitis in UK Holsteins. Page 121 in Proc. 16th Symp. 8th Conf. Lameness in Ruminants, Rotorua, New Zealand. ConferenceNZ, Rotorua, New Zealand.

Sprecher, D. J., D. E. Hostetler, and J. B. Kaneene. 1997. A lameness scoring system that uses posture and gait to predict dairy cattle reproductive performance. Theriogenology 47:1179-1187.

Wells, S. J., L. P. Garber, and B. A. Wagner. 1999. Papillomatous digital dermatitis and associated risk factors in US dairy herds. Prev. Vet. Med. 38:11-24.

Yano, T. 2010. Identification of candidate pathogens of papillomatous digital dermatitis in dairy cattle from quantitative 16S rRNA clonal analysis. Vet. Microbiol. 143:352-362. 